\title{
4-(Trifluoromethoxy)phenyl-Containing Polymers as Promising Anodic Materials for Electrochromic Devices
}

\author{
Wen-Hsin Wang ${ }^{1}$, Jui-Cheng Chang ${ }^{1,2}$, Pei-Ying Lee ${ }^{1}$, Yuan-Chung Lin ${ }^{3}$ and Tzi-Yi Wu ${ }^{1, *(1)}$ \\ 1 Department of Chemical and Materials Engineering, National Yunlin University of Science and Technology, \\ Yunlin 64002, Taiwan; winnie53035@yahoo.com.tw (W.-H.W.); d700215@gmail.com (J.-C.C.); \\ leepeiying1018@gmail.com (P.-Y.L.) \\ 2 Bachelor Program in Interdisciplinary Studies, National Yunlin University of Science and Technology, \\ Yunlin 64002, Taiwan \\ 3 Institute of Environmental Engineering, National Sun Yat-Sen University, Kaohsiung 80424, Taiwan; \\ yclin@faculty.nsysu.edu.tw \\ * Correspondence: wuty@gemail.yuntech.edu.tw; Tel.: +886-5-534-2601 (ext. 4626)
}

Received: 26 November 2020; Accepted: 16 December 2020; Published: 18 December 2020

\begin{abstract}
Three 4-(trifluoromethoxy)phenyl-based polydithienylpyrroles (PTTPP, P(TTPP-co-DTC), and P(TTPP-co-DTP)) were synthesized electrochemically and their electrochromic behaviors were characterized. The introduction of electron withdrawing trifluoromethoxy unit in the side chain of polydithienylpyrroles (PSNS) decreases the HOMO and LUMO energy levels of PSNS. PTTPP film displays three various colors (grayish-yellow at $0 \mathrm{~V}$, grayish-blue at $1.0 \mathrm{~V}$, and bluish-violet at $1.4 \mathrm{~V}$ ) from reduced to oxidized states. The optical contrast of PTTPP, P(TTPP-co-DTC), and P(TTPP-co-DTP) electrodes are $24.5 \%$ at $1050 \mathrm{~nm}, 49.0 \%$ at $916 \mathrm{~nm}$, and $53.8 \%$ at $1302 \mathrm{~nm}$, respectively. The highest $\eta$ of the PTTPP electrode is $379.64 \mathrm{~cm}^{2} \mathrm{C}^{-1}$ at $1050 \mathrm{~nm}$. Three ECDs based on PTTPP, P(TTPP-co-DTC), or P(TTPP-co-DTP) as anodic film and PProDOT-Et ${ }_{2}$ as cathodic film were fabricated. PTTPP/PProDOT-Et ${ }_{2}$ ECD showed high transmittance change $(35.7 \%$ at $588 \mathrm{~nm})$ and high $\eta\left(890.96 \mathrm{~cm}^{2} \cdot \mathrm{C}^{-1}\right.$ at $\left.588 \mathrm{~nm}\right)$. P(TTPP-co-DTC)/PProDOT-Et $t_{2}$ and P(TTPP-co-DTP)/PProDOT-Et ${ }_{2}$ ECDs showed high transmittance change, rapid response time, adequate open circuit memory, and good electrochemical redox stability. Based on these findings, this work provides novel insights for appropriate design of high transmittance change and high efficient multi-colored electrochromic polymers.
\end{abstract}

Keywords: electrochemical polymerization; transmittance; spectroelectrochemistry; electrochromic switching

\section{Introduction}

Electrochromic materials tune their colors reversibly upon applying various potentials or undergoing a redox process. To date, organic and inorganic electrochromic materials have received much interest owing to their probable utilizations in auto-dimming mirror, smart windows of architectures, energy storage devices, etc. [1-3]. Compared to inorganic electrochromic materials, organic electrochromic materials have the benefits of ease of electrochemical and chemical synthesis, large coloration efficiency, rapid electrochromic switching, low onset oxidation voltage, and satisfactory long-term cycling stability $[4,5]$.

In general, conjugated polymers and bipyridinium salts (viologens) are familiar organic electrochromic materials. Polythiophene, polypyrrole, polycarbazole, polyaniline, polyindole, and PEDOT are the most commonly used polymeric materials in electrochemical devices [6-11]. 
According to these conjugated polymers, PProDOT-Et ${ }_{2}$, a derivative of PEDOT, shows prominent performances as a cathodic polymer in ECDs [12]. Polypyrrole and polycarbazole were reported as anodic polymers in ECDs owing to their good hole transporting stability, attractive optical performances, and excellent electroactive characters. Polythiophenes are widely used as electrochromic electrodes due to their good reversible redox behaviors, large conductivity, and large color contrast between reduced and oxidized states [13]. However, the oxidized potentials of non-modified polythiophenes are $1.5 \mathrm{~V}$ (vs. $\mathrm{Ag} / \mathrm{AgCl}$ ) [14]. PSNS consists of two thiophene rings and a pyrrole ring; the strong electron-donating pyrrole unit decreases the oxidized potentials of polymers to $0.7 \mathrm{~V}$ vs. $\mathrm{Ag} / \mathrm{AgCl}$ [15].

Moreover, a trifluoromethoxy $\left(\mathrm{CF}_{3} \mathrm{O}-\right)$ substituent is an electron-withdrawing group, the incorporation of a trifluoromethoxy substituent on the side chain of PSNS decreases the LUMO/HOMO energy levels of PSNS slightly. The electrochromic properties will vary with the change of LUMO/HOMO energy levels.

This paper reports the electrosynthesis and electrochromic properties of three polydithienylpyrrole derivatives (PTTPP, P(TTPP-co-DTC), and P(TTPP-co-DTP)). For a 3,6-di(2-thienyl)carbazole unit, the incorporation of thiophene rings at 3,6-locations of carbazole unit leads to a red shift of the optical UV-Vis band and diminishes the band gap [16]. Dithieno[3,2-b:2', $\left.3^{\prime}-\mathrm{d}\right]$ pyrrole (DTP) is an electron-donating group with a coplanar fused-heterocycle configuration, which assists to increase the polymer backbone planarity and narrow the band gap between conduction band and valence band [17]. The copolymerization of different monomers gives rise to desirable combinations of electrochromic behaviors presented in homopolymers. Therefore, we also synthesized DTC- and DTP-containing copolymers. In addition, dual-type polymer ECDs were prepared using PTTPP, P(TTPP-co-DTC), or P(TTPP-co-DTP) as the anodic material, PProDOT-Et ${ }_{2}$ as the cathodic material, and an ionic liquid-containing electrolyte as the separation layer. Optical and electrochemical characterizations of PTTPP film, P(TTPP-co-DTC) film, P(TTPP-co-DTP) film, PTTPP/PProDOT-Et ${ }_{2}$ ECD, P(TTPP-co-DTC)/PProDOT-Et ${ }_{2}$ ECD, and P(TTPP-co-DTP)/PProDOT-Et ${ }_{2}$ ECD were explored systematically using UV-Vis spectra and electrochromic switching techniques. The cycling stability between the oxidized and reduced states is a crucial factor for ECDs $[18,19]$. Optical memory responds to the energy depletion during the long-term manipulations of ECDs [20,21]. Accordingly, the dual-type polymer ECDs were further explored for optical memory and electrochemical cycling stability.

\section{Experimental}

\subsection{Materials}

Starting chemicals and solvents in this study were bought from TCI, Aldrich, Alfa Aesar, and employed as received. DTC, ProDOT-Et ${ }_{2}$, and $\left[\mathrm{EPI}^{+}\right]\left[\mathrm{TFSI}^{-}\right]$were synthesized on the basis of previously published methods [22-24]. The scheme of 4-(trifluoromethoxy)phenyl-containing SNS derivative (TTPP) is shown in Figure 1. PTTPP, P(TTPP-co-DTC), P(TTPP-co-DTP), and PProDOT-Et ${ }_{2}$ electrodes were polymerized potentiostatically at $0.9 \mathrm{~V}$ on glass substrates.
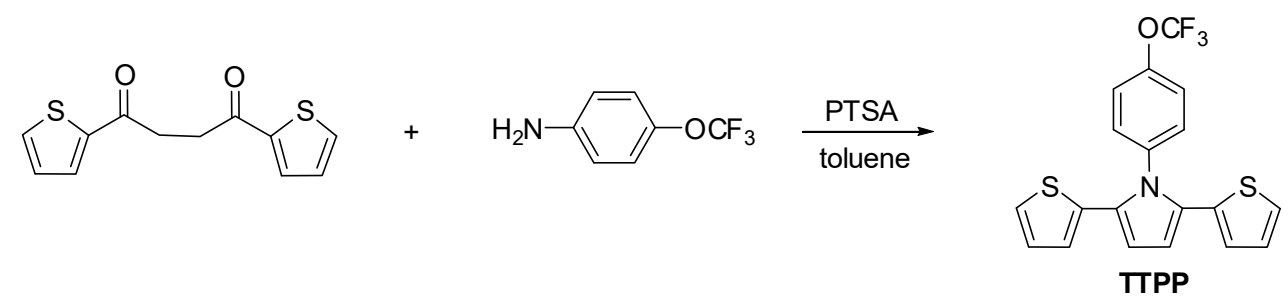

Figure 1. The synthetic scheme of 4-(trifluoromethoxy)phenyl-containing SNS derivative.

\subsection{Preparation of 2,5-Di(Thiophen-2-yl)-1-(4-(Trifluoromethoxy)Phenyl)Pyrrole (TTPP)}

A mixture of 4-(trifluoromethoxy)aniline (0.744 g, $4.2 \mathrm{mmol}), 1,4$-di(2'-thienyl)-1,4-butadione ( $0.35 \mathrm{~g}, 1.4 \mathrm{mmol}), 12 \mathrm{~mL}$ toluene, and $7 \mathrm{mg}$ p-toluenesulfonic acid was stirred for $36 \mathrm{~h}$ under 
reflux. Subsequently, toluene was removed, and the unrefined product was refined using column chromatography. Yield: $63 \% .{ }^{1} \mathrm{H}-\mathrm{NMR}\left(700 \mathrm{MHz}, \mathrm{DMSO}-d_{6}\right): \delta 7.51(\mathrm{~d}, 4 \mathrm{H}$, phenyl-H and Th-H), $7.34(\mathrm{~d}, 2 \mathrm{H}$, phenyl-H), 6.91 (d, 2H, Th-H), $6.72(\mathrm{dd}, 2 \mathrm{H}, \mathrm{Th}-\mathrm{H}), 6.59$ (d, 2H, Py-H). Elem. Anal. Calcd. for $\mathrm{C}_{19} \mathrm{H}_{12} \mathrm{~F}_{3} \mathrm{NOS}_{2}$ : C, 58.30\%; H, 3.09\%; N, 3.58\%. Found: $\mathrm{C}, 58.35 \% ; \mathrm{H}, 3.06 \%$; N, 3.45\%.

\subsection{Electrochemical and Electrochromic Characterizations}

The electrochemical properties of as-prepared PTTPP, P(TTPP-co-DTC), and P(TTPP-co-DTP) electrodes deposited on glass substrates were characterized using an electrochemical analyzer (CHI6081E, CH Instruments, Austin, TX, USA). Electrochromic properties of PTTPP film, P(TTPP-co-DTC) film, P(TTPP-co-DTP) electrode, PTTPP/PProDOT-Et ${ }_{2}$ ECD, P(TTPP-co-DTC)/PProDOT-Et $t_{2}$ ECD, and P(TTPP-co-DTP)/PProDOT-Et ${ }_{2}$ ECD were characterized by a spectrophotometer (V-670 JASCO Pfungstadt, Germany).

\subsection{Fabrication of ECDs}

The preparations of $\left.\left[\mathrm{EPI}^{+}\right]\left[\mathrm{TFSI}^{-}\right]\right) / \mathrm{PVdF} \mathrm{HFP}$ composite electrolytes were represented previously [25]. The anodic layers (PTTPP, P(TTPP-co-DTC), and P(TTPP-co-DTP)) were prepared potentiostatically onto glass substrates at $+0.9 \mathrm{~V}$, respectively, whereas the cathodic layer (PProDOT-Et ${ }_{2}$ ) was deposited potentiostatically onto glass substrate at $+1.4 \mathrm{~V}$. The electrodes were isolated by the composite electrolyte. The electrode area of as-prepared ECDs was $1.8 \mathrm{~cm}^{2}$.

\section{Results and Discussion}

\subsection{Electrochemical Preparation of Electrodes}

Figure 2 displays the synthetic schemes of PTTPP, P(TTPP-co-DTC), and P(TTPP-co-DTP) and Figure 3 shows the potentiodynamic measurements of neat TTPP, DTC, and DTP and their mixtures (TTPP+DTC and TTPP+DTP) in a solution. As the potentiodynamic measurements scanned continued, the anodic and cathodic peaks in Figure 3 increased stage by stage, implying PTTPP, PDTC, PDTP, $\mathrm{P}$ (TTPP-co-DTC), and P(TTPP-co-DTP) films were electrodeposited onto the ITO substrates. The onset oxidized potentials ( $E_{\text {onset }}$ ) of TTPP, DTC, and DTP were $0.68,0.82$, and $0.80 \mathrm{~V}$, respectively. The $E_{\text {onset }}$ disparities of TTPP vs. DTC and TTPP vs. DTP were smaller than $0.15 \mathrm{~V}$, inferring the copolymerization of TTPP vs. DTC and TTPP vs. DTP was workable [26]. Furthermore, the $E_{\text {onset }}$ of TTPP was smaller than those of DTP and DTC, implying that a 4-(trifluoromethoxy)phenyl-based dithienylpyrrole was more susceptible to oxidation than either the DTP or DTC. The oxidized peaks of PTTPP, P(TTPP-co-DTC), and P(TTPP-co-DTP), as shown in Figure 3, locate at 0.77, 1.55 , and $0.96 \mathrm{~V}$, respectively, whereas the reduced peaks of PTTPP, P(TTPP-co-DTC), and P(TTPP-co-DTP) situate at 0.17, 0.15, and $0.34 \mathrm{~V}$, respectively.
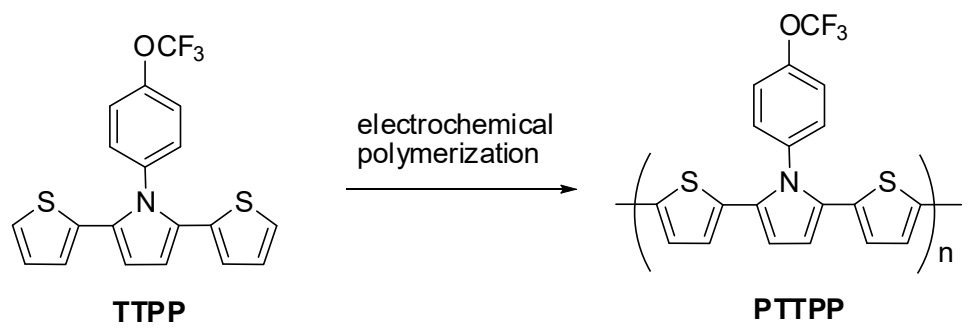

Figure 2. Cont. 
<smiles>FC(F)(F)Oc1ccc(-n2c(-c3cccs3)ccc2-c2cccs2)cc1</smiles>

TTPP<smiles>FC(F)(F)Oc1ccc(-n2c(-c3cccs3)ccc2-c2cccs2)cc1</smiles>

TTPP

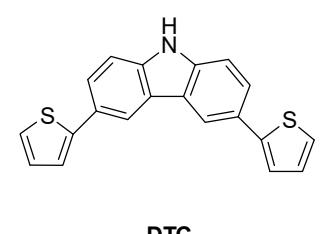

DTC

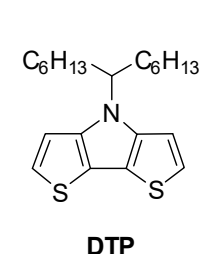

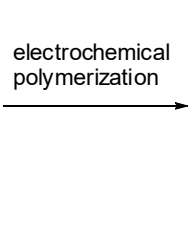

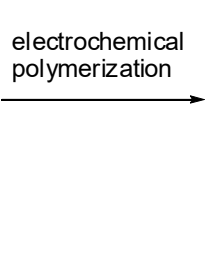

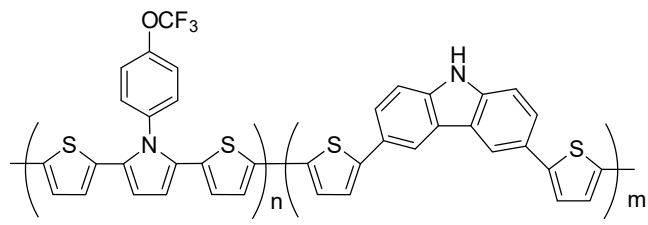

P(TTPP-co-DTC)

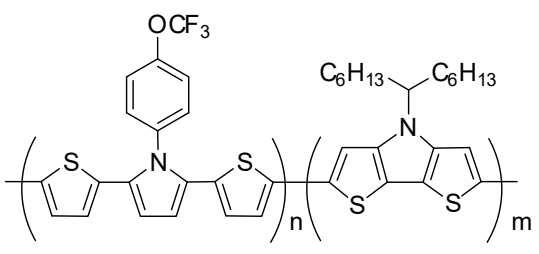

P(TTPP-co-DTP)

Figure 2. The electrosynthetic schemes of three 4-(trifluoromethoxy)phenyl-containing PSNS.

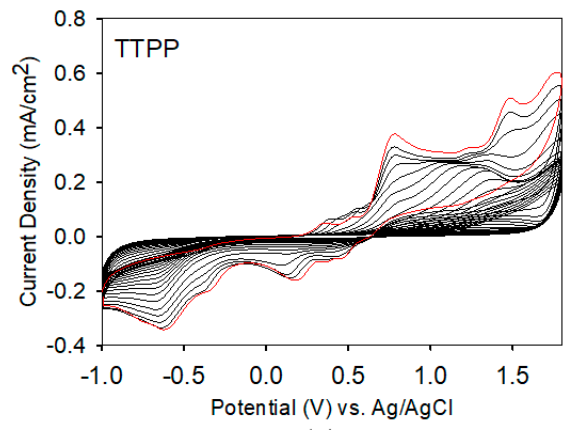

(a)

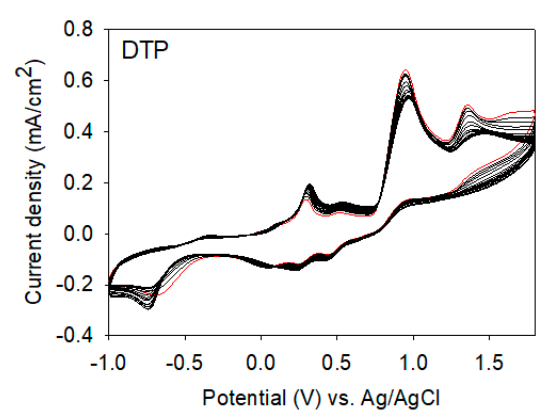

(c)

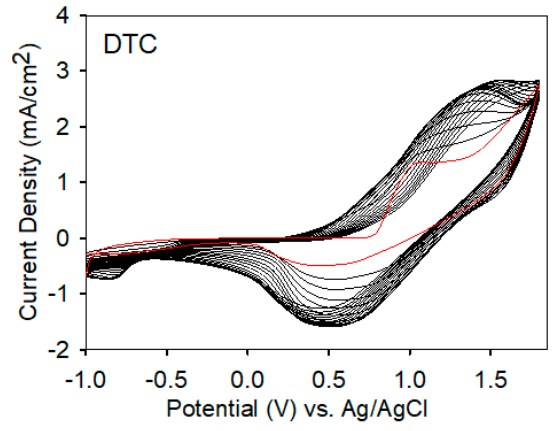

(b)

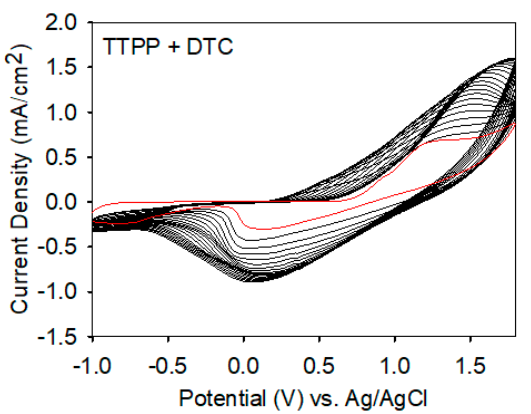

(d)

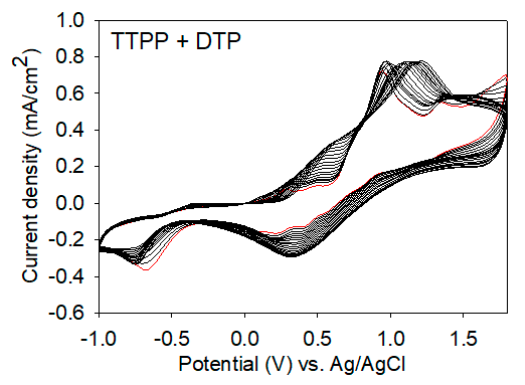

(e)

Figure 3. Cyclic curves of: (a) $2 \mathrm{mM}$ TTPP; (b) $2 \mathrm{mM} \mathrm{DTC}$; (c) $2 \mathrm{mM} \mathrm{DTP}$; (d) $2 \mathrm{mM}$ TTPP + $2 \mathrm{mM}$ DTC; and (e) $2 \mathrm{mM}$ TTPP $+2 \mathrm{mM}$ DTP in $0.1 \mathrm{M}$ lithium perchlorate/acetonitrile. The red line indicates the first cycle. 
Figure 4a-c displays the cyclic voltammograms of PTTPP, P(TTPP-co-DTC), and P(TTPP-co-DTP) electrodes, respectively, at scan rates between 25 and $200 \mathrm{mV} \mathrm{s}^{-1}$, while Figure $4 \mathrm{~d}-\mathrm{f}$ displays their corresponding charts of peak current and sweep velocity. The peak current densities raise linearly with raising scan rate, inferring PTTPP, P(TTPP-co-DTC), and P(TTPP-co-DTP) are well clung onto ITO surface and the redox reactions are non-diffusion limited processes [27].

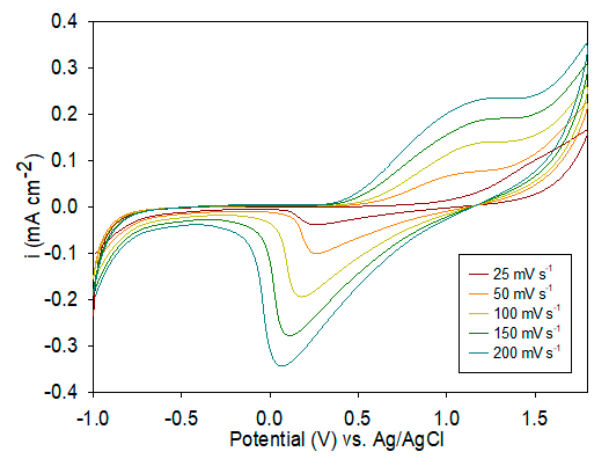

(a)

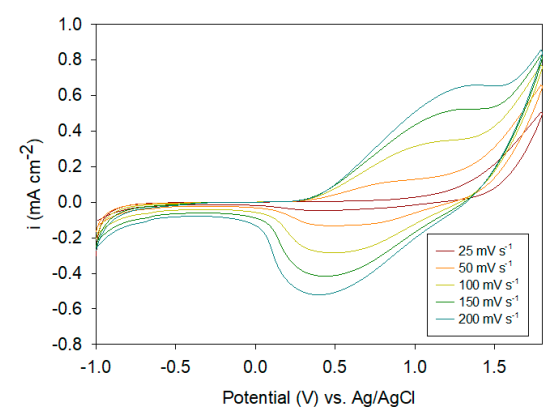

(b)

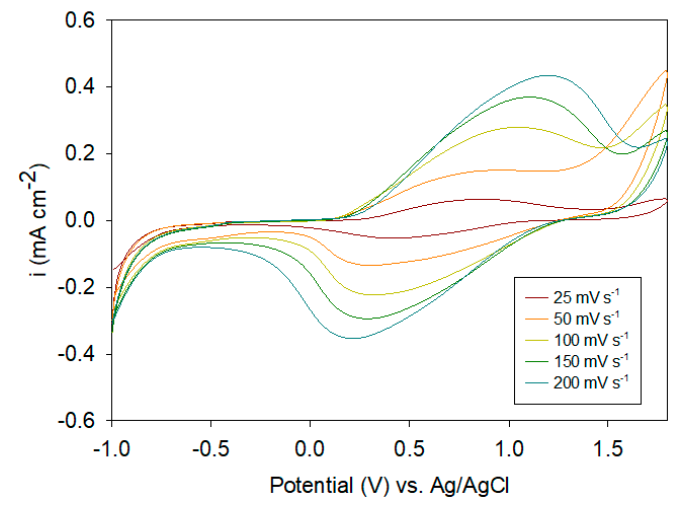

(c)

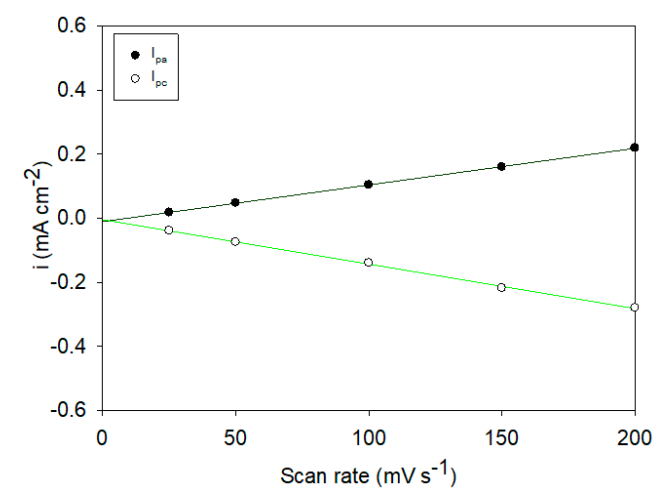

(d)

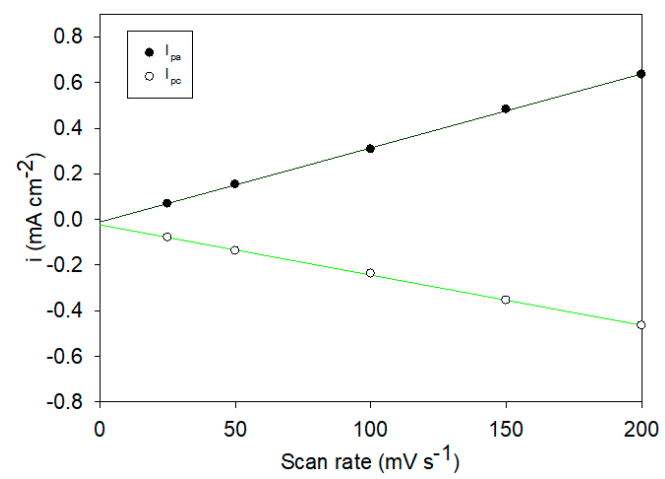

(e)

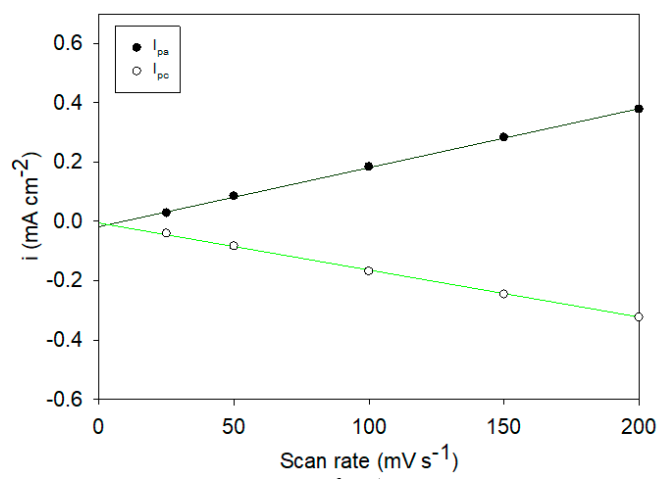

(f)

Figure 4. CV plots of: (a) PTTPP; (b) P(TTPP-co-DTC); and (c) P(TTPP-co-DTP) electrodes at different scan rates in $0.1 \mathrm{M}$ lithium perchlorate/acetonitrile. The relationship of $i$ vs. $v$ of: (d) PTTPP; (e) P(TTPP-co-DTC); and (f) P(TTPP-co-DTP) electrodes. 


\subsection{Electrochromic Characterizations of PTTPP, P(TTPP-co-DTC), and P(TTPP-co-DTP)}

As shown in Figure 5, the UV-Vis band of PTTPP film in the reduction state situated at $364 \mathrm{~nm}$. The absorption band of P(TTPP-co-DTC) and P(TTPP-co-DTP) in the reduction state shifted bathochromically relative to PTTPP film. It is worth noting that the absorption band red shift value of P(TTPP-co-DTP) film is larger than that of P(TTPP-co-DTC) film, which can be ascribed to the fused-heterocycle of DTP unit being able to increase the planarity of repeating units in P(TTPP-co-DTP) backbones and diminish polymeric bandgap. As the application of potential increases gradually, the $\pi-\pi^{*}$ transition absorbance peak of PTTPP film begins to diminish, and bipolaron and polaron bands generate at ca. 532 and $1050 \mathrm{~nm}$. Under similar condition, the bipolaron and polaron bands generate at $916 \mathrm{~nm}$ for P(TTPP-co-DTC) film and $1302 \mathrm{~nm}$ for P(TTPP-co-DTP) film. Table 1 displays the electrochromic photoimages of PTTPP, P(TTPP-co-DTC), and P(TTPP-co-DTP) in a solution at different redox potentials; the anodic polymer films display reversible electrochromic phenomena in their neutral and oxidized states. The PTTPP film was grayish-yellow at $0 \mathrm{~V}$, grayish-blue at $1.0 \mathrm{~V}$, and bluish-violet at $1.4 \mathrm{~V}$. Under similar circumstances, $\mathrm{P}(\mathrm{TTPP}-c o-\mathrm{DTC})$ displays obvious color transition with three colors (grayish-green at $0 \mathrm{~V}$, grayish-blue at $1.0 \mathrm{~V}$, and bluish-purple at $1.2 \mathrm{~V}$ ), while P(TTPP-co-DTP) film shows three kinds of colors from reduced to oxidized states (gray at $0 \mathrm{~V}$, grayish-blue at $0.6 \mathrm{~V}$, and blue at $1.2 \mathrm{~V}$ ). The colorimetric values of PTTPP, P(TTPP-co-DTC), and P(TTPP-co-DTP) estimated at different voltages are summarized in Table 1 , and the chromaticity diagrams of PTTPP, P(TTPP-co-DTC), and P(TTPP-co-DTP) in reduced and oxidized state are displayed in Figure 6.

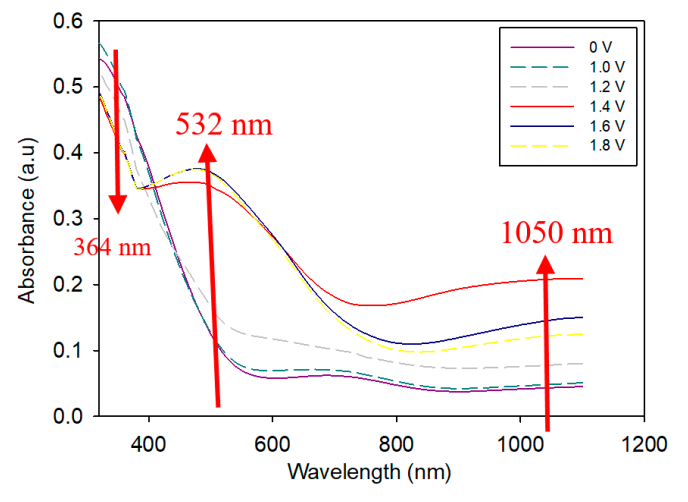

(a)

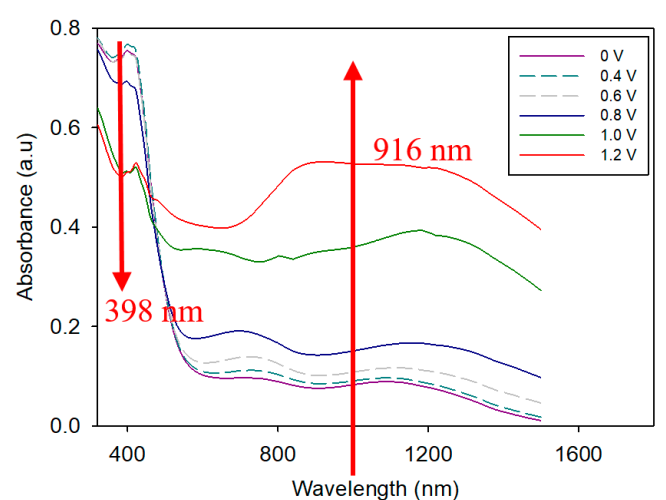

(b)

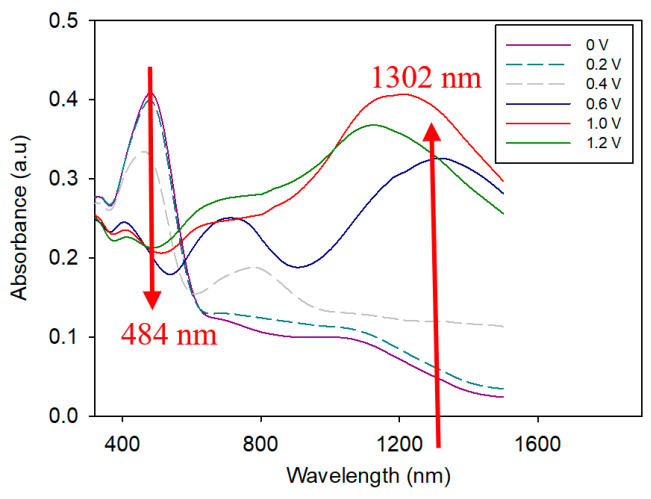

(c)

Figure 5. Absorption spectra of: (a) PTTPP; (b) P(TTPP-co-DTC); and (c) P(TTPP-co-DTP) films at different voltages. 


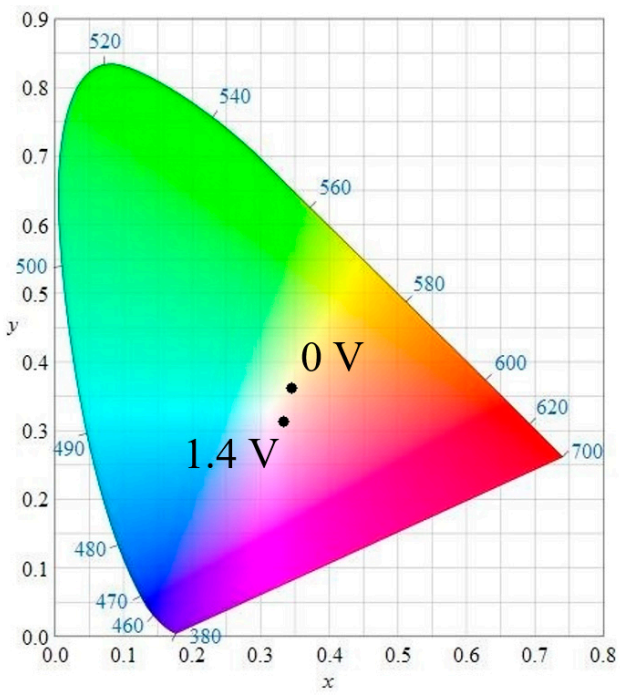

(a)

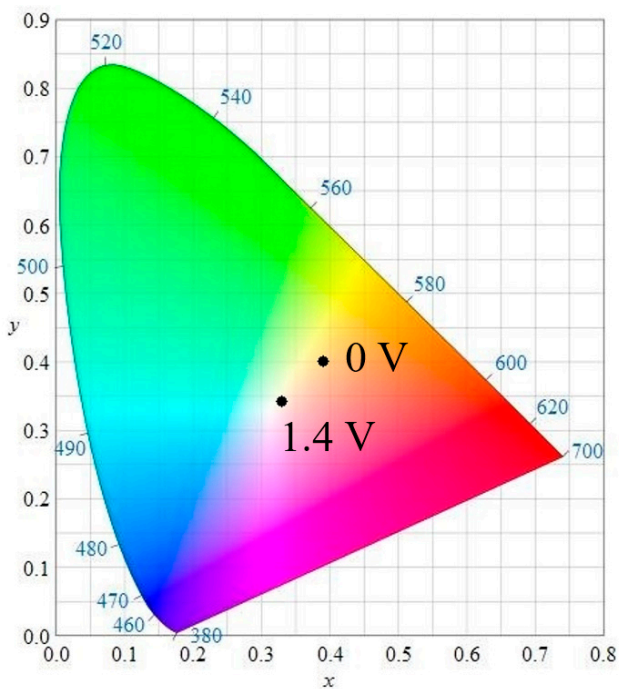

(b)

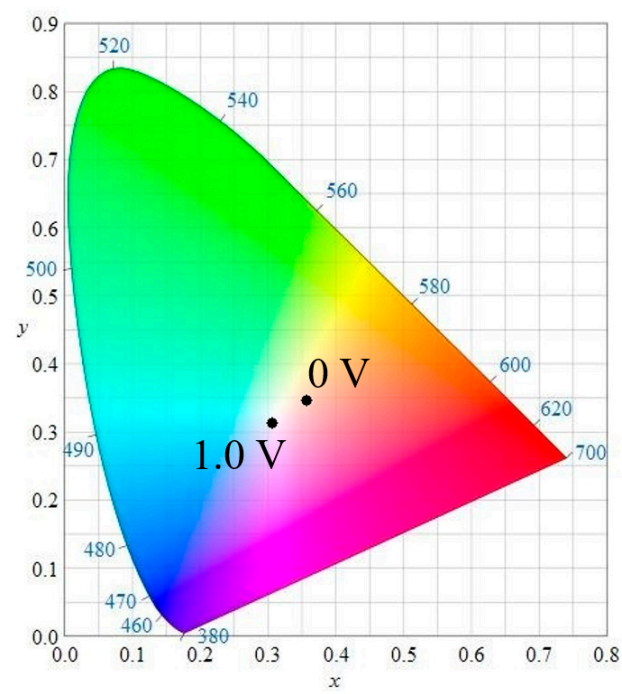

(c)

Figure 6. CIE chromaticity charts of: (a) PTTPP; (b) P(TTPP-co-DTC); and (c) P(TTPP-co-DTP) electrodes in $\left[\mathrm{EPI}^{+}\right]\left[\mathrm{TFSI}^{-}\right]$solution in the oxidized and reduced states.

The band gap $\left(E_{\mathrm{g}}\right)$ of PTTPP film can be determined from the $\lambda_{\text {onset }}$ of UV spectra by Planck equation $\left(E_{\mathrm{g}}=1241 / \lambda_{\text {onset }}\right)$ [28], and it is $2.34 \mathrm{eV}$ (Table 2). The HOMO/LUMO energy levels of PTTPP film were determined using the following equations [29]:

$$
\begin{gathered}
E_{\mathrm{HOMO}}=-\mathrm{e}\left(E_{\text {onset }}+4.8\right)(\text { vs. vacuum }) \\
E_{\mathrm{LUMO}}=E_{\mathrm{HOMO}}+E_{\mathrm{g}}
\end{gathered}
$$

where $E_{\text {onset }}$ is corrected using ferrocene as internal standard. The $E_{\text {HOMO }}$ and $E_{\text {LUMO }}$ of PTTPP film are -4.91 and $-2.52 \mathrm{eV}$, respectively. 
Table 1. Electrochromic behaviors of PSNS.

\begin{tabular}{|c|c|c|c|c|c|}
\hline Polymers & $E / \mathrm{V}$ & Photographs & $L^{*}$ & $a^{*}$ & $b^{*}$ \\
\hline \multirow{3}{*}{ РТТРР } & 0 & & 92.11 & -2.13 & 19.48 \\
\hline & 1.0 & & 91.09 & -2.13 & 17.43 \\
\hline & 1.4 & & 75.4 & 6.8 & 4.77 \\
\hline \multirow{3}{*}{ P(TTPP-co-DTC) } & 0 & & 86.23 & -3.16 & 43.75 \\
\hline & 1.0 & & 71.81 & -3.3 & 9.7 \\
\hline & 1.2 & & 68.18 & -0.87 & 7.17 \\
\hline \multirow{3}{*}{ P(TTPP-co-DTP) } & 0 & & 82.49 & 14.04 & 14.73 \\
\hline & 0.6 & & 87.79 & -4.73 & 3.79 \\
\hline & 1.2 & & 84.85 & -3.03 & -1.81 \\
\hline
\end{tabular}

$L^{*}$ indicates the lightness, $a^{*}$ and $b^{*}$ represent the color channels.

Double step chronoamperometry technique was used to estimate switching performances of PTTPP, P(TTPP-co-DTC), and P(TTPP-co-DTP) [30]. Figure 7 exhibits the time-transmittance profiles of PTTPP, $\mathrm{P}(\mathrm{TTPP}-c o-\mathrm{DTC})$, and $\mathrm{P}(\mathrm{TTPP}-\mathrm{co}$-DTP) with an interval of $5 \mathrm{~s}$ between redox states. The coloration/bleaching time $\left(\tau_{\mathrm{c}}\right.$ and $\left.\tau_{\mathrm{b}}\right)$ of PTTPP, P(TTPP-co-DTC), and P(TTPP-co-DTP) are summarized in Table 3. The $\tau_{\mathrm{c}}$ and $\tau_{\mathrm{b}}$ are estimated at $90 \%$ of whole transmittance variation $\left(T_{90 \%}\right)$. The $\tau_{\mathrm{c}}$ and $\tau_{\mathrm{b}}$ of three polymer films at visible light and near infrared light regions were found to be $1.85-2.49 \mathrm{~s}$ in $\left[\mathrm{EPI}^{+}\right]\left[\mathrm{TFSI}^{-}\right]$solution.

The optical contrast $\Delta T(\%)$ is a crucial parameter for electrochromic materials and devices [31]. The $\Delta T_{\max }$ of PTTPP, P(TTPP-co-DTC), and P(TTPP-co-DTP) are $24.5 \%$ at $1050 \mathrm{~nm}, 49.0 \%$ at $916 \mathrm{~nm}$, and $53.8 \%$ at $1302 \mathrm{~nm}$, respectively, in $\left[\mathrm{EPI}^{+}\right]\left[\mathrm{TFSI}^{-}\right]$solution, indicating copolymers show higher $\Delta T$ than that of PTTPP homopolymer and the introduction of DTP group in the polymeric backbone leads to higher $\Delta T_{\max }$ than that of DTC unit. For the three polymer films, P(TTPP-co-DTP) shows the highest $\Delta T_{\max }(53.8 \%)$ at $1302 \mathrm{~nm}$. As listed in Table 2, the $\Delta T_{\max }$ of P(TTPP-co-DTP) film was larger than those reported for poly(1-co-EDOT) $\left(\Delta T_{\max }=32.9 \%\right.$ at $\left.500 \mathrm{~nm}\right)$ [32], $\mathrm{PBCB}\left(\Delta T_{\max }=44 \%\right.$ at $\left.1000 \mathrm{~nm}\right)$ [33], and $\operatorname{PBCP}\left(\Delta T_{\max }=39 \%\right.$ at $\left.1000 \mathrm{~nm}\right)$ [33]. 


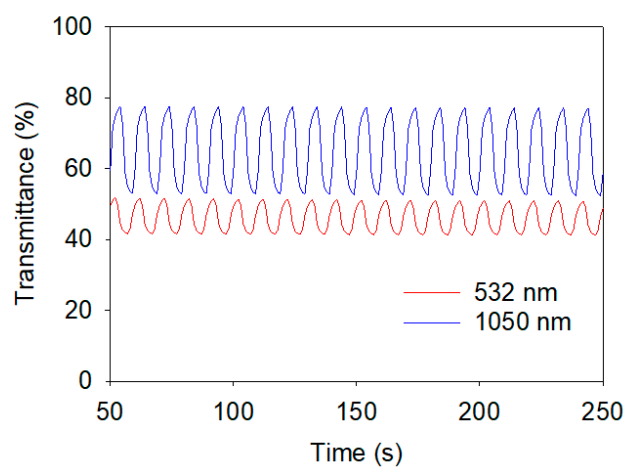

(a)

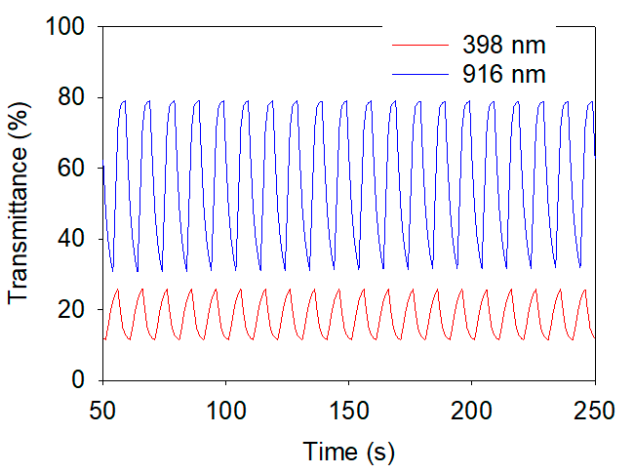

(b)

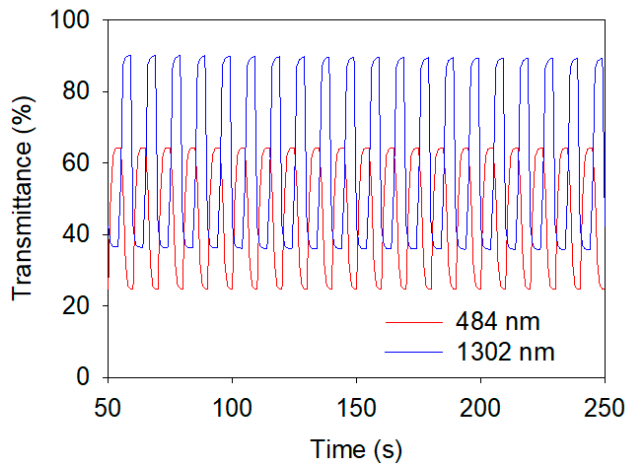

(c)

Figure 7. Transmittance profiles of: (a) PTTPP film at 532 and $1050 \mathrm{~nm}$; (b) P(TTPP-co-DTC) film at 398 and $916 \mathrm{~nm}$; and (c) P(TTPP-co-DTP) film at 484 and $1302 \mathrm{~nm}$ under potentials between 0 and $+1.0 \mathrm{~V}$. Time interval: $5 \mathrm{~s}$.

Table 2. Comparisons of the $\Delta T_{\max }$ and $\eta_{\max }$ for polymers and ECDs.

\begin{tabular}{|c|c|c|c|c|c|}
\hline Polymers and ECDs & $\lambda / \mathrm{nm}$ & $E_{\mathrm{g}} / \mathrm{eV}$ & $\Delta T_{\max } / \%$ & $\Delta \mathrm{OD}_{\max } / \%$ & $\eta_{\max } / \mathrm{cm}^{2} \mathrm{C}^{-1}$ \\
\hline РТTPP & 1050 & 2.34 & 24.5 & 16.5 & 379.64 \\
\hline P(TTPP-co-DTC) & 916 & - & 49.0 & 41.9 & 171.29 \\
\hline P(TTPP-со-DTP) & 1302 & - & 53.8 & 39.5 & 394.82 \\
\hline poly(1-co-EDOT) [32] & 500 & - & 32.9 & - & 173 \\
\hline PBCB [33] & 1000 & 1.77 & 44 & - & 268.43 \\
\hline PBCP [33] & 1000 & 1.74 & 39 & - & 236.18 \\
\hline PTTPP/PProDOT-Et ${ }_{2}$ ECD & 588 & - & 35.7 & 59.4 & 890.96 \\
\hline P(TTPP-co-DTC)/PProDOT-Et ${ }_{2}$ ECD & 590 & - & 42.6 & 56.9 & 512.79 \\
\hline P(TTPP-co-DTP)/PProDOT-Et ${ }_{2}$ ECD & 592 & - & 48.1 & 77.8 & 519.27 \\
\hline P(TTPA-co-EDOT)/PEDOT ECD [34] & 650 & - & 24 & - & 545 \\
\hline P(FPTP-co-EDOT)/PEDOT ECD [35] & 555 & - & 23 & - & - \\
\hline
\end{tabular}


Table 3. Coloring-bleaching kinetics of polymer electrodes and ECDs.

\begin{tabular}{|c|c|c|c|c|c|c|c|c|}
\hline \multirow{2}{*}{ Polymers and ECDs } & \multirow{2}{*}{$\lambda_{\max } / \mathrm{nm}$} & \multirow{2}{*}{ Cycle No. } & \multicolumn{2}{|c|}{$T$} & \multicolumn{3}{|c|}{$\tau\left(T_{90 \%}\right)$} & \multirow{2}{*}{$\begin{array}{l}\text { Stability } \\
\text { (100 cycles) }\end{array}$} \\
\hline & & & $T_{\mathrm{b}} / \%$ & $T_{\mathrm{c}} / \%$ & $\Delta T / \%$ & $\tau_{\mathrm{c} / \mathrm{s}}$ & $\tau_{\mathrm{b}} / \mathbf{s}$ & \\
\hline \multirow{2}{*}{$\begin{array}{c}\text { PTTPP in } \\
{\left[\mathrm{EPI}^{+}\right]\left[\mathrm{TFSI}^{-}\right]}\end{array}$} & 532 & 1 & 51.9 & 41.7 & 10.2 & 2.32 & 1.99 & - \\
\hline & - & 50 & 49.6 & 40.7 & 8.9 & 2.33 & 1.86 & - \\
\hline- & - & 100 & 48.7 & 40.4 & 8.3 & 2.29 & 2.07 & $81.4 \%$ \\
\hline- & 1050 & 1 & 77.7 & 53.2 & 24.5 & 2.41 & 1.87 & - \\
\hline- & - & 50 & 76.4 & 51.9 & 24.5 & 2.41 & 1.89 & - \\
\hline- & - & 100 & 75.3 & 52.4 & 22.9 & 2.39 & 1.95 & $93.5 \%$ \\
\hline \multirow{2}{*}{$\begin{array}{c}\mathrm{P}\left(\mathrm{TTPP}^{-c o-D T C}\right) \text { in } \\
{\left[\mathrm{EPI}^{+}\right]\left[\mathrm{TFSI}^{-}\right]}\end{array}$} & 398 & 1 & 26.0 & 11.5 & 14.5 & 2.34 & 2.18 & - \\
\hline & - & 50 & 25.6 & 11.4 & 14.2 & 2.41 & 2.28 & - \\
\hline- & - & 100 & 25.4 & 11.4 & 14.0 & 2.30 & 2.33 & $96.6 \%$ \\
\hline- & 916 & 1 & 79.2 & 30.2 & 49.0 & 2.03 & 2.42 & - \\
\hline- & - & 50 & 78.7 & 33.1 & 45.6 & 1.94 & 2.46 & - \\
\hline- & - & 100 & 78.6 & 35.4 & 43.2 & 1.96 & 2.49 & $88.2 \%$ \\
\hline \multirow{3}{*}{$\begin{array}{c}\mathrm{P}\left(\mathrm{TTPP}^{-c o-D T P}\right) \text { in } \\
{\left[\mathrm{EPI}^{+}\right]\left[\mathrm{TFSI}^{-}\right]}\end{array}$} & 484 & 1 & 64.3 & 24.7 & 39.6 & 1.85 & 1.88 & - \\
\hline & - & 50 & 64.0 & 24.5 & 39.5 & 1.87 & 1.86 & - \\
\hline & - & 100 & 63.9 & 24.4 & 39.5 & 1.97 & 1.91 & $99.7 \%$ \\
\hline- & 1302 & 1 & 90.1 & 36.3 & 53.8 & 1.96 & 1.92 & - \\
\hline- & - & 50 & 88.9 & 35.5 & 53.4 & 1.94 & 2.02 & - \\
\hline- & - & 100 & 88.7 & 35.4 & 53.3 & 1.92 & 1.89 & $99.1 \%$ \\
\hline \multirow{3}{*}{$\begin{array}{c}\text { PTTPP/ } \\
\text { PProDOT-Et }_{2} \text { ECD }\end{array}$} & 588 & 1 & 47.9 & 12.2 & 35.7 & 0.96 & 0.97 & - \\
\hline & - & 50 & 47.8 & 12.8 & 35.0 & 0.94 & 0.94 & - \\
\hline & - & 100 & 48.0 & 13.9 & 34.1 & 0.99 & 0.92 & $95.5 \%$ \\
\hline P(TTPP-co-DTC)/ & 590 & 1 & 58.3 & 15.7 & 42.6 & 0.99 & 0.94 & - \\
\hline PProDOT-Et $_{2}$ ECD & - & 50 & 58.0 & 15.6 & 42.4 & 0.93 & 0.91 & - \\
\hline- & - & 100 & 56.8 & 15.7 & 41.1 & 0.97 & 0.90 & $96.5 \%$ \\
\hline P(TTPP-co-DTP)/ & 592 & 1 & 57.7 & 9.6 & 48.1 & 0.94 & 0.88 & - \\
\hline PProDOT-Et $_{2}$ ECD & - & 50 & 56.9 & 9.7 & 47.2 & 0.92 & 0.90 & - \\
\hline- & - & 100 & 56.7 & 10.1 & 46.6 & 0.87 & 0.94 & $96.9 \%$ \\
\hline
\end{tabular}

The coloration efficiency $(\eta)$ can be estimated by the following equations [36]:

$$
\begin{gathered}
\Delta \mathrm{OD}=\log \left(T_{\mathrm{b}} / T_{\mathrm{c}}\right) \\
\eta=\Delta \mathrm{OD} / Q_{\mathrm{d}}
\end{gathered}
$$

where $\Delta \mathrm{OD}, T_{\mathrm{b}}$, and $T_{\mathrm{c}}$ denote the variation of the optical density, the transmittance of bleaching state, and the transmittance of coloring state, respectively. The $\eta_{\max }$ of PTTPP, P(TTPP-co-DTC), and P(TTPP-co-DTP) films are $379.64 \mathrm{~cm}^{2} / \mathrm{C}$ at $1050 \mathrm{~nm}, 171.29 \mathrm{~cm}^{2} / \mathrm{C}$ at $916 \mathrm{~nm}$, and $394.82 \mathrm{~cm}^{2} / \mathrm{C}$ at $1302 \mathrm{~nm}$, respectively. P(TTPP-co-DTP) film shows the highest $\eta_{\max }$. PTTPP film shows higher $\eta$ than those reported for poly(1-co-EDOT) $\left(\eta=173 \mathrm{~cm}^{2} / \mathrm{C}\right.$ at $\left.500 \mathrm{~nm}\right)$ [32], $\mathrm{PBCB}\left(\eta=268.43 \mathrm{~cm}^{2} / \mathrm{C}\right.$ at $1000 \mathrm{~nm})[33]$, and PBCP $\left(\eta=236.18 \mathrm{~cm}^{2} / \mathrm{C}\right.$ at $\left.1000 \mathrm{~nm}\right)[33]$.

\subsection{Spectroelectrochemical Characterization of Electrochromic Devices}

Dual-type complementary ECDs consisted of an anodic layer (PTTPP, P(TTPP-co-DTC), or P(TTPP-co-DTP)), a cathodic layer (PProDOT-Et 2 ), and an ionic liquid/PVdF-HFP electrolyte were constructed and their optical performances were measured. As presented in Figure 8a, PTTPP/PProDOT-Et ${ }_{2}$ ECD displayed an absorption peak at ca. $367 \mathrm{~nm}$ at $-0.4 \mathrm{~V}$, which could be ascribed to the $\pi-\pi^{*}$ transition of PTTPP in the reduced state. Under the situation, the cathodic layer was light blue in its oxidized state, and PTTPP/PProDOT-Et ${ }_{2}$ ECD was gray at $-0.4 \mathrm{~V}$. However, the $\pi-\pi^{*}$ transition peak of PTTPP decreased gradually and a peak emerged at $588 \mathrm{~nm}$ progressively with increasing 
voltage. The PTTPP/PProDOT-Et ${ }_{2}$ ECD was deep blue at $1.2 \mathrm{~V}$ (Table 4). Under similar circumstances,

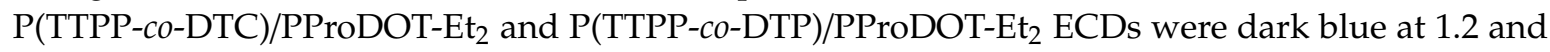
$1.4 \mathrm{~V}$, respectively. The CIE chromaticity charts of PTTPP/PProDOT-Et ${ }_{2}, \mathrm{P}\left(\mathrm{TTPP}-\mathrm{co}\right.$-DTC)/PProDOT-Et ${ }_{2}$, and P(TTPP-co-DTP)/PProDOT-Et $t_{2}$ ECDs at bleached and colored voltages are displayed in Figure 9.

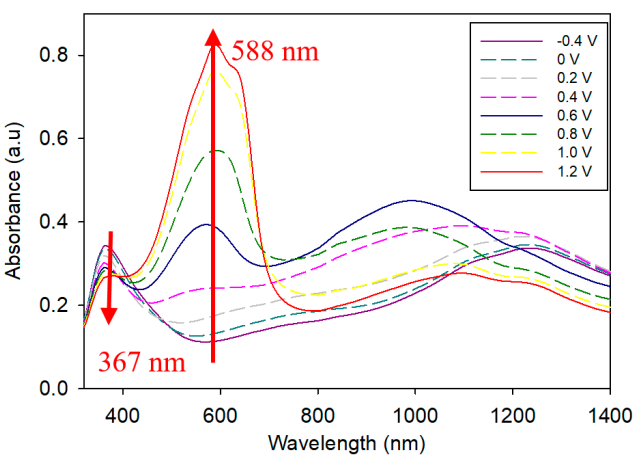

(a)

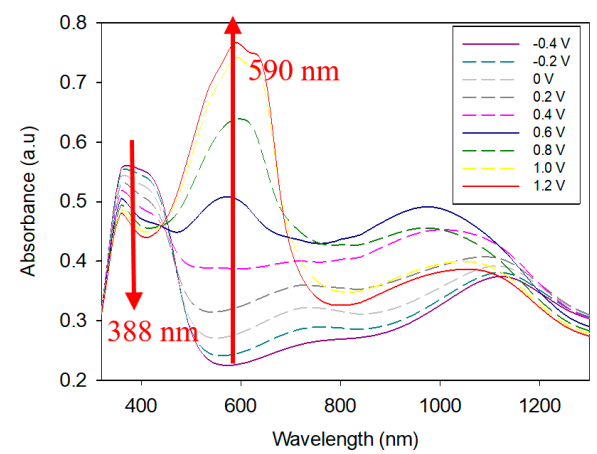

(b)

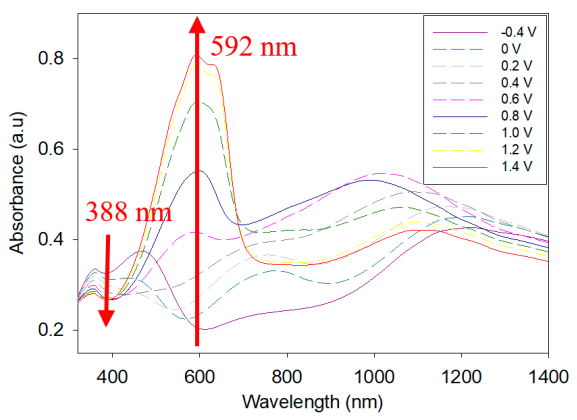

(c)

Figure 8. UV plots of: (a) PTTPP/PProDOT-Et ${ }_{2}$; (b) P(TTPP-co-DTC)/PProDOT-Et $t_{2}$; and (c) P(TTPP-coDTP)/PProDOT-Et ${ }_{2}$ ECDs at various voltages.

Figure 10a-c reveals the time-transmittance charts of PTTPP/PProDOT-Et ${ }_{2}, \mathrm{P}\left(\mathrm{TTPP}-\mathrm{co}^{-}\right.$ DTC)/PProDOT-Et ${ }_{2}$, and P(TTPP-co-DTP)/PProDOT-Et ${ }_{2}$ ECDs, respectively, and the $\Delta T, \Delta \mathrm{OD}$, and coloration efficiency of PTTPP/PProDOT-Et ${ }_{2}, \mathrm{P}(\mathrm{TTPP}-\mathrm{co}-\mathrm{DTC}) / \mathrm{PProDOT}_{\mathrm{E}} \mathrm{E}_{2}$, and P(TTPPco-DTP)/PProDOT-Et $t_{2}$ ECDs are listed in Table 2. The $\Delta T_{\max }$ of PTTPP/PProDOT-Et $t_{2}, \mathrm{P}(\mathrm{TTPP}-\mathrm{co}-$ DTC)/PProDOT-Et ${ }_{2}$, and P(TTPP-co-DTP)/PProDOT-Et ${ }_{2}$ ECDs were $35.7 \%$ at $588 \mathrm{~nm}, 42.6 \%$ at $590 \mathrm{~nm}$, and $48.1 \%$ at $592 \mathrm{~nm}$, respectively. In other aspects, the $\eta_{\max }$ of PTTPP/PProDOT-Et ${ }_{2}$, P(TTPP-co-DTC)/PProDOT-Et $t_{2}$, and P(TTPP-co-DTP)/PProDOT-Et ${ }_{2}$ ECDs were calculated to be $890.96 \mathrm{~cm}^{2} / \mathrm{C}$ at $588 \mathrm{~nm}, 512.79 \mathrm{~cm}^{2} / \mathrm{C}$ at $590 \mathrm{~nm}$, and $519.27 \mathrm{~cm}^{2} / \mathrm{C}$ at $592 \mathrm{~nm}$, respectively. The $\Delta T_{\max }$ of PTTPP/PProDOT-Et ${ }_{2}, \mathrm{P}\left(\mathrm{TTPP}-\mathrm{co}\right.$-DTC)/PProDOT-Et $\mathrm{P}_{2}$, and P(TTPP-co-DTP)/PProDOT-Et ${ }_{2}$ ECDs are larger than those of P(TTPA-co-EDOT)/PEDOT ECD $\left(\Delta T_{\max }=24 \%\right.$ at $\left.650 \mathrm{~nm}\right)$ [35] and $\mathrm{P}\left(\right.$ FPTP-co-EDOT)/PEDOT ECD $\left(\Delta T_{\max }=23 \%\right.$ at $\left.555 \mathrm{~nm}\right)$ [36]. Moreover, PTTPP/PProDOT-Et ${ }_{2}$ ECD shows higher $\eta_{\max }$ than that reported for P(TTPA-co-EDOT)/PEDOT ECD $\left(\eta_{\max }=545 \mathrm{~cm}^{2} / \mathrm{C}\right.$ at $650 \mathrm{~nm})[35]$.

The coloration/bleaching time of ECDs estimated after various cycles are shown in Table 3 . The $\tau_{\mathrm{c}}$ and $\tau_{\mathrm{b}}$ of PTTPP/PProDOT-Et $t_{2}, \mathrm{P}\left(\right.$ TTPP-co-DTC)/PProDOT-Et ${ }_{2}$, and P(TTPP-co-DTP)/PProDOT-Et ${ }_{2}$ ECDs are in the range of $0.87-0.99 \mathrm{~s}$. The coloration/bleaching times of PTTPP/PProDOT-Et ${ }_{2}$, P(TTPP-co-DTC)/PProDOT-Et ${ }_{2}$, and P(TTPP-co-DTP)/PProDOT-Et $t_{2}$ ECDs were faster than those of PTTPP, $\mathrm{P}(\mathrm{TTPP}-c o-\mathrm{DTC})$, and $\mathrm{P}(\mathrm{TTPP}-\mathrm{co}$-DTP) electrodes, indicating the ECDs changed color from bleached to colored state more quickly than PTTPP, P(TTPP-co-DTC), and P(TTPP-co-DTP) films in a solution. 
Table 4. Photoimages and $L^{*}, a^{*}$, and $b^{*}$ values of ECDs.

\begin{tabular}{|c|c|c|c|c|c|}
\hline ECDs & $E / \mathrm{V}$ & Photoimages & $L^{*}$ & $a^{*}$ & $b^{*}$ \\
\hline \multirow{5}{*}{ PTTPP/PProDOT-Et ${ }_{2}$} & -0.4 & & 87.38 & -1.64 & 8.91 \\
\hline & 0 & & 85.98 & -2.28 & 5.47 \\
\hline & 0.6 & & 72.62 & 4.5 & -10.44 \\
\hline & 0.8 & & 65.31 & 4.11 & -19.6 \\
\hline & 1.2 & & 56.03 & 5.65 & -30.98 \\
\hline \multirow{5}{*}{ P(TTPP-co-DTC)/PProDOT-Et ${ }_{2}$} & 0 & & 81.05 & -7.69 & 19.29 \\
\hline & 0.4 & & 73.43 & -3.39 & 6.91 \\
\hline & 0.6 & & 67.22 & -1.06 & -2.54 \\
\hline & 0.8 & & 61.72 & -0.31 & -10.81 \\
\hline & 1.2 & & 59 & 1.65 & -15.34 \\
\hline \multirow{5}{*}{ P(TTPP-co-DTP)/PProDOT-Et ${ }_{2}$} & -0.4 & & 81.78 & 8.71 & 5.4 \\
\hline & 0 & & 85.15 & -1.07 & 4.43 \\
\hline & 0.6 & & 76.9 & 1.94 & -10.44 \\
\hline & 0.8 & & 70.37 & 3.26 & -19.12 \\
\hline & 1.4 & $\theta$ & 57.72 & 5.9 & -35.2 \\
\hline
\end{tabular}




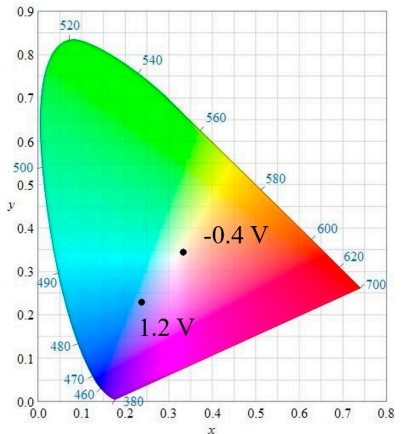

(a)

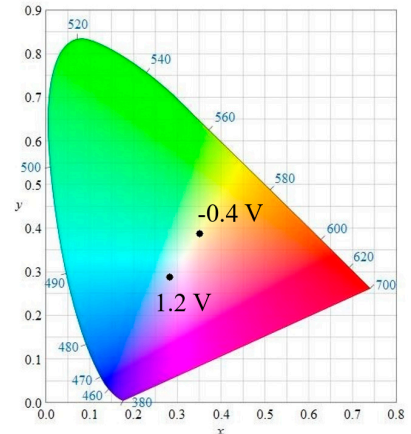

(b)

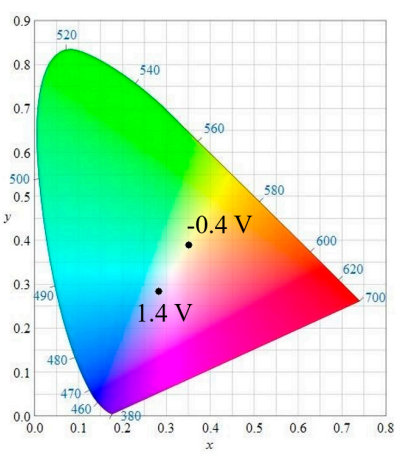

(c)

Figure 9. CIE chromaticity charts of: (a) PTTPP/PProDOT-Et 2 ; (b) P(TTPP-co-DTC)/PProDOT-Et ${ }_{2}$; and (c) P(TTPP-co-DTP)/PProDOT-Et ${ }_{2}$ ECDs at bleached and colored states.

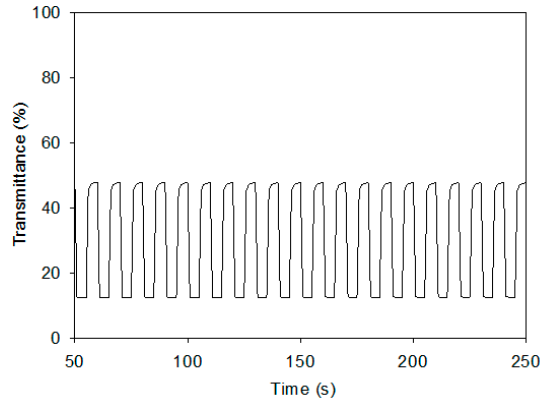

(a)

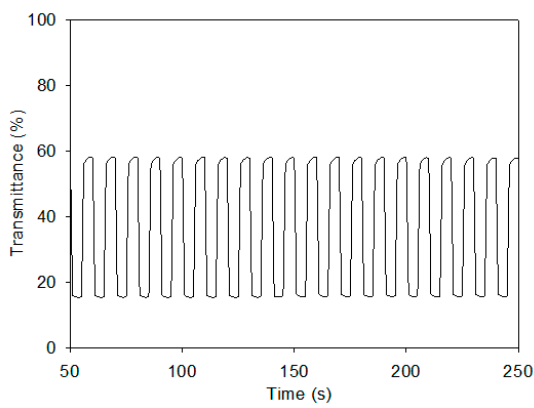

(b)

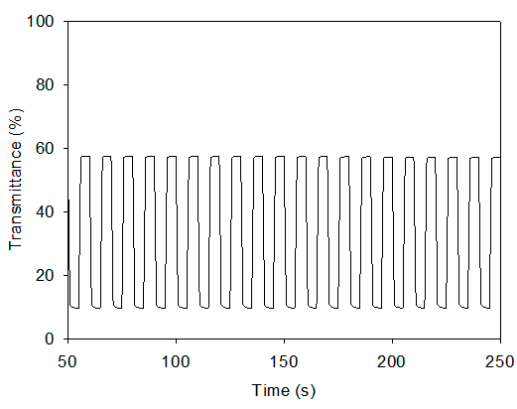

(c)

Figure 10. Transmittance of: (a) PTTPP/PProDOT-Et ${ }_{2}$ ECD at $588 \mathrm{~nm}$ (between -0.2 and $+1.0 \mathrm{~V}$ ); (b) P(TTPP-co-DTC)/PProDOT-Et 2 ECD at $590 \mathrm{~nm}$ (between -0.4 and $1.4 \mathrm{~V}$ ); and (c) P(TTPPco-DTP)/PProDOT-Et ${ }_{2}$ ECD at $592 \mathrm{~nm}$ (between -0.4 and $1.4 \mathrm{~V}$ ). 


\subsection{Cycling Stability and Optical Memory of Electrochromic Devices}

The cycling stability of PTTPP/PProDOT-Et ${ }_{2}$, P(TTPP-co-DTC)/PProDOT-Et ${ }_{2}$, and P(TTPPco-DTP)/PProDOT-Et ${ }_{2}$ ECDs were monitored by cyclic voltammetry measurements at voltages between -1.0 and $+1.8 \mathrm{~V}$. As displayed in Figure $11,95.2 \%, 97.2 \%$, and $92.7 \%$, respectively, of electroactivity were preserved for PTTPP/PProDOT-Et ${ }_{2}, \mathrm{P}$ (TTPP-co-DTC)/PProDOT-Et ${ }_{2}$, and P(TTPP-co-DTP)/PProDOT-Et ${ }_{2}$ ECDs after the 500th cycle, and $88.9 \%, 96.1 \%$, and 91.3\%, respectively, of electroactivity were preserved after the 1000th cycle, indicating PTTPP/PProDOT-Et ${ }_{2}$, P(TTPP-co-DTC)/PProDOT-Et ${ }_{2}$, and P(TTPP-co-DTP)/PProDOT-Et $t_{2}$ ECDs displayed a sufficient redox cycling stability.

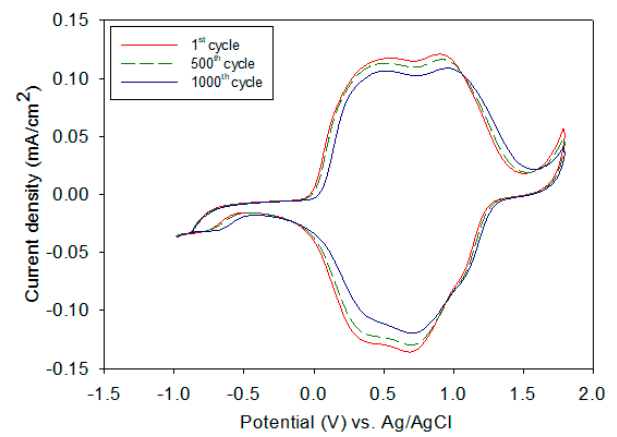

(a)

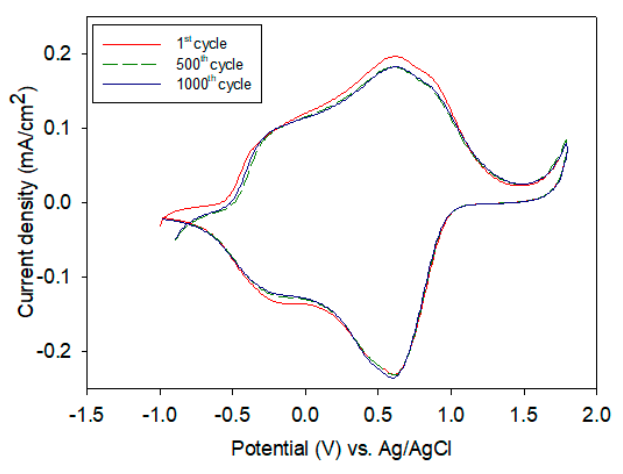

(b)

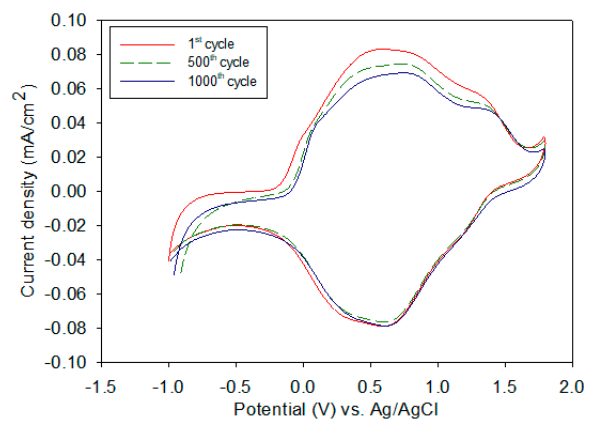

(c)

Figure 11. CV plots of: (a) PTTPP/PProDOT-Et $t_{2}$ (b) P(TTPP-co-DTC)/PProDOT-Et ${ }_{2}$; and (c) P(TTPPco-DTP)/PProDOT-Et ${ }_{2}$ ECDs.

The optical memory of PTTPP/PProDOT-Et ${ }_{2}$, P(TTPP-co-DTC)/PProDOT-Et $t_{2}$, and P(TTPPco-DTP)/PProDOT-Et ${ }_{2}$ ECDs were measured at $588-592 \mathrm{~nm}$ by applying voltages at coloring state $(+1.0$ or $+1.4 \mathrm{~V})$ and bleaching state $(-0.4$ or $-0.2 \mathrm{~V})$ for $1 \mathrm{~s}$ for each $200 \mathrm{~s}$ time interval. As displayed in Figure 12, PTTPP/PProDOT-Et ${ }_{2}$, P(TTPP-co-DTC)/PProDOT-Et ${ }_{2}$, and P(TTPP-co-DTP)/PProDOT-Et ${ }_{2}$ ECDs presented sufficient open circuit memory at coloring state (the variation of transmittance was less than $5 \%$ ) and bleaching state (transmittance change was less than 1.5\%), verifying PTTPP/PProDOT-Et ${ }_{2}$,

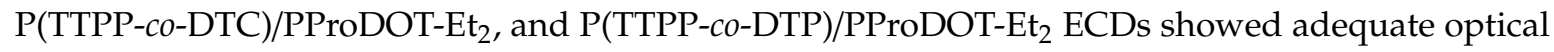
memory at coloring and bleaching states. 


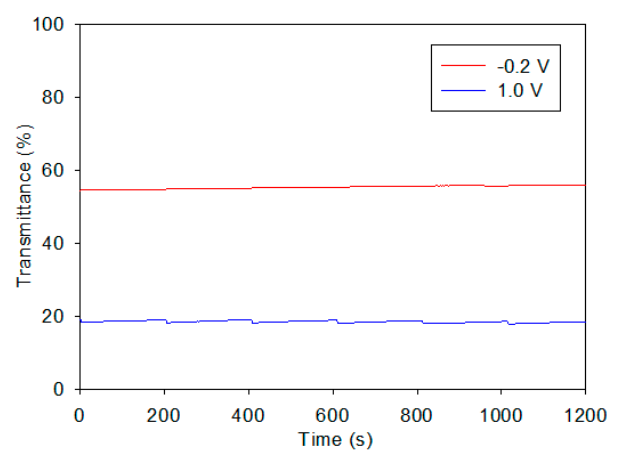

(a)

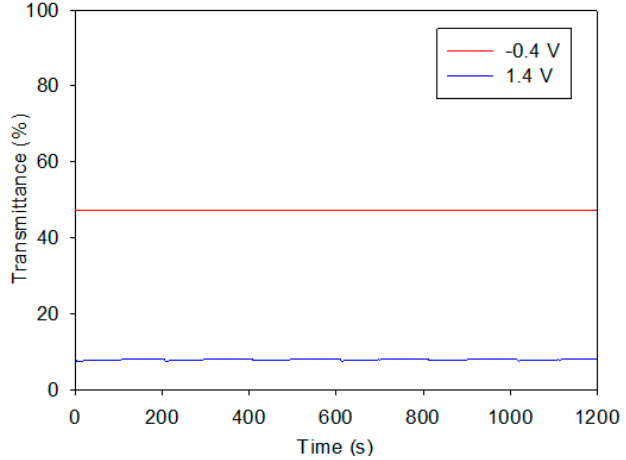

(b)

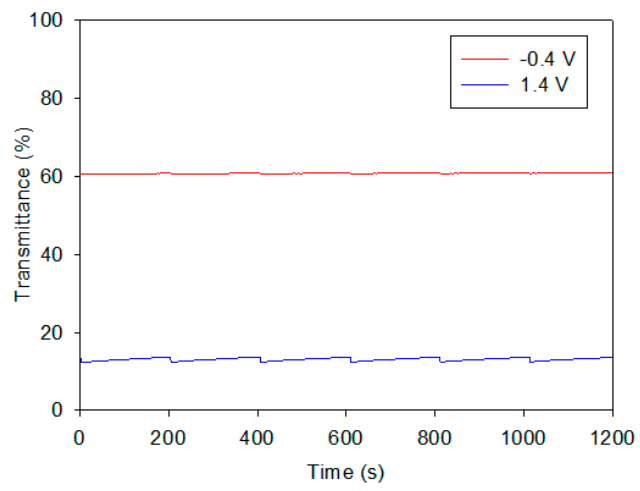

(c)

Figure 12. Optical memory effect of: (a) PTTPP/PProDOT-Et ${ }_{2}$ ECD measured at $588 \mathrm{~nm}$; (b) P(TTPPco-DTC)/PProDOT-Et ${ }_{2}$ ECD measured at $590 \mathrm{~nm}$; and (c) P(TTPP-co-DTP)/PProDOT-Et ${ }_{2}$ ECD measured at $592 \mathrm{~nm}$.

\section{Conclusions}

TTPP was synthesized and its corresponding homopolymers (PTTPP) and copolymers (P(TTPP-co-DTC) and P(TTPP-co-DTP)) were prepared. The anodic polymer films display reversible electrochromic phenomena in their neutral and oxidized states. The PTTPP film was grayish-yellow at $0 \mathrm{~V}$, grayish-blue at $1.0 \mathrm{~V}$, and bluish-violet at $1.4 \mathrm{~V}$, whereas $\mathrm{P}(\mathrm{TTPP}-\mathrm{co}-\mathrm{DTP})$ film showed color changes from reduced to oxidized states (gray at $0 \mathrm{~V}$, grayish-blue at $0.6 \mathrm{~V}$, and blue at $1.2 \mathrm{~V}$ ). Colorless-to-colorful switching investigations of anodic films show that $\mathrm{P}(\mathrm{TTPP}-\mathrm{co}-\mathrm{DTP})$ film has high $\Delta T_{\max }(53.8 \%$ at $1302 \mathrm{~nm})$ and PTTPP film has high $\eta\left(379.64 \mathrm{~cm}^{2} / \mathrm{C}\right.$ at $\left.1050 \mathrm{~nm}\right)$. P(TTPP-co-DTP)/PProDOT-Et ${ }_{2}$ ECD exhibits high $\Delta T_{\max }(48.1 \%$ at $592 \mathrm{~nm})$ and a sufficient cycling stability, whereas PTTPP/PProDOT-Et 2 ECD displays high $\eta\left(890.96 \mathrm{~cm}^{2} / \mathrm{C}\right.$ at $\left.588 \mathrm{~nm}\right)$ and adequate optical memory at coloring and bleaching states, implying PTTPP, P(TTPP-co-DTC), and P(TTPP-co-DTP) films are promising candidates as anodic electrochromic materials for potential applications in motorcycle helmet-visors, electrochromic goggles, electrochromic display devices, and auto-dimming car mirror.

Author Contributions: Conceptualization, W.-H.W. and T.-Y.W.; methodology, W.-H.W.; formal analysis, W.-H.W. and T.-Y.W.; investigation, W.-H.W., J.-C.C. and P.-Y.L.; resources, Y.-C.L.; data curation, W.-H.W. and T.-Y.W.; writing_-original draft preparation, T.-Y.W.; writing—review and editing, T.-Y.W.; project administration, T.-Y.W. and Y.-C.L.; funding acquisition, T.-Y.W. and Y.-C.L. All authors have read and agreed to the published version of the manuscript.

Funding: This research was funded by Ministry of Science and Technology of Republic of China, Grant No. 108-2221-E-224-049-MY3.

Conflicts of Interest: The authors declare no conflict of interest. 


\section{References}

1. Cai, G.F.; Wang, J.X.; Lee, P.S. Next-generation multifunctional electrochromic devices. Acc. Chem. Res. 2016, 49, 1469-1476. [CrossRef]

2. Beaujuge, P.M.; Reynolds, J.R. Color control in $\pi$-conjugated organic polymers for use in electrochromic devices. Chem. Rev. 2010, 110, 268-320. [CrossRef]

3. Rosseinsky, D.R.; Mortimer, R.J. Electrochromic systems and the prospects for devices. Adv. Mater. 2001, 13, 783-793. [CrossRef]

4. Mortimer, R.J. Electrochromic materials. Annu. Rev. Mater. Res. 2011, 41, 241-268. [CrossRef]

5. Kuo, C.-W.; Wu, B.-W.; Chang, J.-K.; Chang, J.-C.; Lee, L.-T.; Wu, T.-Y.; Ho, T.-H. Electrochromic devices based on poly(2,6-di(9H-carbazol-9-yl)pyridine)-type polymer films and PEDOT-PSS. Polymers 2018, 10, 604. [CrossRef] [PubMed]

6. Kuo, C.W.; Wu, T.Y.; Fan, S.C. Applications of poly(indole-6-carboxylic acid-co-2,2'-bithiophene) films in high-contrast electrochromic devices. Coatings 2018, 8, 102. [CrossRef]

7. Hsiao, S.H.; Lin, S.W. Electrochemical synthesis of electrochromic polycarbazole films from $N$-phenyl-3,6-bis(N-carbazolyl)carbazoles. Polym. Chem. 2016, 7, 198-211. [CrossRef]

8. Kuo, C.W.; Hsieh, T.H.; Hsieh, C.K.; Liao, J.W.; Wu, T.Y. Electrosynthesis and characterization of four electrochromic polymers based on carbazole and indole-6-carboxylic acid and their applications in high-contrast electrochromic devices. J. Electrochem. Soc. 2014, 161, D782-D790. [CrossRef]

9. Camurlu, P. Polypyrrole derivatives for electrochromic applications. RSC Adv. 2014, 4, 55832-55845. [CrossRef]

10. Hacioglu, S.O.; Yiğit, D.; Ermis, E.; Soylemez, S.; Güllü, M.; Toppare, L. Syntheses and electrochemical characterization of low oxidation potential nitrogen analogs of pedot as electrochromic materials. J. Electrochem. Soc. 2016, 163, E293-E299. [CrossRef]

11. Kuo, C.W.; Chen, B.K.; Li, W.B.; Tseng, L.Y.; Wu, T.Y.; Tseng, C.G.; Chen, H.R.; Huang, Y.C. Effects of supporting electrolytes on spectroelectrochemical and electrochromic properties of polyaniline-poly(styrene sulfonic acid) and poly(ethylenedioxythiophene)-poly(styrene sulfonic acid)-based electrochromic device. J. Chin. Chem. Soc. 2014, 61, 563-570. [CrossRef]

12. Otley, M.T.; Alamer, F.A.; Zhu, Y.; Singhaviranon, A.; Zhang, X.; Li, M.; Kumar, A.; Sotzing, G.A. Acrylated poly(3,4-propylenedioxythiophene) for enhancement of lifetime and optical properties for single-layer electrochromic devices. ACS Appl. Mater. Interfaces 2014, 6, 1734-1739. [CrossRef]

13. Ouyang, M.; Wang, G.H.; Zhang, Y.J.; Hua, C.; Zhang, C. Multicolored electrochromic copolymer based on 1,4-di(thiophen-3-yl)benzene and 3,4-ethylenedioxythiophene. J. Electroanal. Chem. 2011, 653, 21-26. [CrossRef]

14. Atılgan, N.; Cihaner, A.; Önal, A.M. Electrochromic performance and ion sensitivity of a terthienyl based fluorescent polymer. React. Funct. Polym. 2010, 70, 244-250. [CrossRef]

15. Chang, K.H.; Wang, H.P.; Wu, T.Y.; Sun, I.W. Optical and electrochromic characterizations of four 2,5-dithienylpyrrole-based conducting polymer films. Electrochim. Acta 2014, 119, 225-235. [CrossRef]

16. Kuo, C.W.; Chang, J.C.; Huang, Y.T.; Chang, J.K.; Lee, L.T.; Wu, T.Y. Applications of copolymers consisting of 2,6-di(9H-carbazol-9-yl)pyridine and 3,6-di(2-thienyl)carbazole units as electrodes in electrochromic devices. Materials 2019, 12, 1251. [CrossRef] [PubMed]

17. Zhang, W.; Li, J.; Zhang, B.; Qin, J. Highly fluorescent conjugated copolymers containing dithieno $\left[3,2-b: 2^{\prime}, 3^{\prime}-d\right]$ pyrrole. Macromol. Rapid Commun. 2008, 29, 1603-1608. [CrossRef]

18. Su, Y.S.; Chang, J.C.; Wu, T.Y. Applications of three dithienylpyrroles-based electrochromic polymers in high-contrast electrochromic devices. Polymers 2017, 9, 114. [CrossRef]

19. Guzela, M.; Karatasbz, E.; Ak, M. Synthesis and fluorescence properties of carbazole based asymmetric functionalized star shaped polymer. J. Electrochem. Soc. 2017, 164, H49-H55. [CrossRef]

20. Kuo, C.W.; Lee, P.Y. Electrosynthesis of copolymers based on 1,3,5-tris( $N$-carbazolyl)benzene and 2,2'-bithiophene and their applications in electrochromic devices. Polymers 2017, 9, 518. [CrossRef]

21. Kuo, C.W.; Wu, T.Y.; Huang, M.W. Electrochromic characterizations of copolymers base on 4,4'-bis(N-carbazole)-1,1'-biphenyl and indole-6-carboxylic acid and their application in electrochromic devices. J. Taiwan Inst. Chem. Eng. 2016, 68, 481-488. [CrossRef]

22. Su, Y.S.; Wu, T.Y. Three carbazole-based polymers as potential anodically coloring materials for high-contrast electrochromic devices. Polymers 2017, 9, 284. [CrossRef] 
23. Welsh, D.M.; Kumar, A.; Meijer, E.W.; Reynolds, J.R. Enhanced contrast ratios and rapid switching in electrochromics based on poly(3,4-propylenedioxythiophene) derivatives. Adv. Mater. 1999, 11, 1379-1382. [CrossRef]

24. Wu, T.Y.; Chen, B.K.; Hao, L.; Lin, K.F.; Sun, I.W. Thermophysical properties of a room temperature ionic liquid (1-methyl-3-pentyl-imidazolium hexafluorophosphate) with poly(ethylene glycol). J. Taiwan Inst. Chem. Eng. 2011, 42, 914-921. [CrossRef]

25. Wu, T.Y.; Liao, J.W.; Chen, C.Y. Electrochemical synthesis, characterization and electrochromic properties of indan and 1,3-benzodioxole-based poly(2,5-dithienylpyrrole) derivatives. Electrochim. Acta 2014, 150, 245-262. [CrossRef]

26. Wu, T.Y.; Su, Y.S.; Chang, J.C. Dithienylpyrrole- and tris[4-(2-thienyl)phenyl]amine-containing copolymers as promising anodic layers in high-contrast electrochromic devices. Coatings 2018, 8, 164. [CrossRef]

27. Oğuztûrk, H.E.; Tirkeș, S.; Önal, A.M. Electrochemical synthesis of new conjugated polymers based on carbazole and furan units. J. Electroanal. Chem. 2015, 750, 1-8. [CrossRef]

28. Wu, T.Y.; Tsao, M.H.; Chen, F.L.; Su, S.G.; Chang, C.W.; Wang, H.P.; Lin, Y.C.; Ou-Yang, W.C.; Sun, I.W. Synthesis and characterization of organic dyes containing various donors and acceptors. Int. J. Mol. Sci. 2010, 11, 329-353. [CrossRef]

29. Tsao, M.H.; Wu, T.Y.; Wang, H.P.; Sun, I.W.; Su, S.G.; Lin, Y.C.; Chang, C.W. An efficient metal free sensitizer for dye-sensitized solar cells. Mater. Lett. 2011, 65, 583-586. [CrossRef]

30. Kuo, C.W.; Chang, J.K.; Lin, Y.C.; Wu, T.Y.; Lee, P.Y.; Ho, T.H. Poly(tris(4-carbazoyl-9-ylphenyl)amine)/three poly(3,4-ethylenedioxythiophene) derivatives in complementary high-contrast electrochromic devices. Polymers 2017, 9, 543. [CrossRef]

31. Hsiao, S.H.; Liao, Y.C. Facile synthesis of electroactive and electrochromic triptycene poly(ether-imide)s containing triarylamine units via oxidative electro-coupling. Polymers 2017, 9, 497. [CrossRef] [PubMed]

32. Algi, M.P.; Öztaş, Z.; Tirkes, S.; Cihaner, A.; Algi, F. A new electrochromic copolymer based on dithienylpyrrole and EDOT. Org. Electron. 2013, 14, 1094-1102. [CrossRef]

33. Hu, B.; Luo, W.; Jin, L.; Liu, Z.; Wang, M.; Zhou, L.; Li, C. Electrochemical and spectroelectrochemical properties of poly(carbazole-EDOT)s derivatives functionalized with benzonitrile and phthalonitrile units. ECS J. Solid State Sci. Technol. 2016, 5, P21-P26. [CrossRef]

34. Cheng, X.; Zhao, J.; Fu, Y.; Cui, C.; Zhang, X. Electrosynthesis and characterization of a multielectrochromic copolymer of tris[4 -(2-thienyl)phenyl]amine with 3,4-ethylenedioxythiophene. J. Electrochem. Soc. 2013, 160, G6-G13. [CrossRef]

35. Turkarslan, O.; Ak, M.; Tanyeli, C.; Akhmedov, I.M.; Toppare, L. Enhancing electrochromic properties of conducting polymers via copolymerization: Copolymer of 1-(4-fluorophenyl)-2,5-di(thiophen-2-yl)-1Hpyrrole with 3,4-ethylene dioxythiophene. J. Polym. Sci. Pol. Chem. 2007, 45, 4496-4503. [CrossRef]

36. Feng, F.; Kong, L.; Du, H.; Zhao, J.; Zhang, J. Donor-acceptor-type copolymers based on 3,4-propylenedioxy-thiophene and 5,6-difluorobenzotriazole: Synthesis and electrochromic properties. Polymers 2018, 10, 427. [CrossRef]

Publisher's Note: MDPI stays neutral with regard to jurisdictional claims in published maps and institutional affiliations.

(C) 2020 by the authors. Licensee MDPI, Basel, Switzerland. This article is an open access article distributed under the terms and conditions of the Creative Commons Attribution (CC BY) license (http://creativecommons.org/licenses/by/4.0/). 